IP $\rightleftharpoons$ B

\title{
Distribuição diamétrica e principais espécies arbóreas presentes nos ecossistemas de Miombo, Mopane e Mecrusse em Moçambique
}

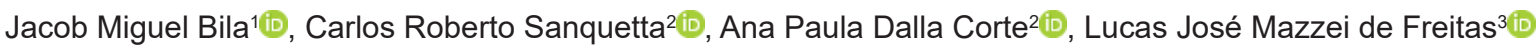 \\ ${ }^{1}$ Instituto de Investigação Agrária de Moçambique, Avenida das FPLM, 2698, CP 3658, Maputo, Moçambique \\ ${ }^{2}$ Universidade Federal do Paraná, Av. Lothário Meissner, 632, CEP 80210-170, Curitiba, PR, Brasil \\ ${ }^{2}$ Embrapa Amazônia Oriental, Trav. Dr. Eneas Pinheiro Marco, CP. 48, CEP 66095-903, Belém, PA, Brasil
}

\section{"Autor correspondente: \\ jacobila@yahoo.com.br \\ Termos para indexação: \\ Manejo de povoamento florestal \\ Ecologia vegetal \\ Função de weibull}

Index terms:

Forest of management stands

Plant ecology

Weibull function

\section{Histórico do artigo:}

Recebido em 25/10/2017

Aprovado em 01/07/2018

Publicado em 19/10/2018

doi: 10.4336/2018.pfb.38e201701523
Resumo - Os recursos florestais em Moçambique desempenham um papel importante na economia nacional, não só para a satisfação das necessidades básicas das comunidades locais, como também para o abastecimento da indústria florestal e para exportação. Este trabalho teve por objetivo comparar o desempenho das funções densidade de probabilidade nos ecossistemas de Miombo, Mopane e Mecrusse no ajuste da distribuição diamétrica e análise fitossociológica destes ecossistemas. Foram usadas as funções densidade de probabilidade de Weibull 3P, Gama e Beta. A estrutura horizontal foi analisada, para identificação das espécies com maior participação na distribuição diamétrica nos ecossistemas em estudo. A função de Weibull 3P foi aderente para os três ecossistemas, a função de Gama e Beta apresentaram aderência nos ecossistemas de Miombo e Mopane. Os três ecossistemas apresentam diferentes espécies predominantes, sendo Julbernardia globiflora, Brachystegia spiciformes, Brachystegia boemii, Pterocarpus angolensis e Ptelliopsis myrtifolia; em Miombo; Colophospermum mopane, Boscia mossambicensis, Adansonia digitada, Afzelia quansensis e Kirkia acuminata, em Mopane, e em Mecrusse Androstachys johnsonii foi a única espécie encontrada. Em função das diferenças observadas, recomenda-se que o manejo florestal seja orientado pelas particularidades de cada ecossistema.

\section{Diametric distribution and main species in Miombo, Mopane and Mecrusse ecosystems in Mozambique}

\begin{abstract}
Forest resources in Mozambique play an important role in the national economy, not only meeting basic needs of local communities, but also to supply the forestry industry and to export. This work aimed to evaluate the performance of probability density functions in the adjustment of the diametric distribution and phytosociological analysis through the horizontal structure of Miombo, Mopane and Mecrusse ecosystems. We adjusted the function Weibull 3P, Gama and Beta functions. The horizontal structure was analysed to verify the species with greater participation in the diametric distribution in the studied ecosystems. Weibull 3P function presented better adherence in all studied ecosystems; Gama and Beta function showed adherence in Miombo and Mopane. The three ecosystems presented different predominant species: in Miombo they were Julbernardia globiflora, Brachystegia spiciformes, Brachystegia boemii, Pterocarpus angolensis and Ptelliopsis myrtifolia; in Mopane Colophospermum mopane, Boscia mossambicensis, Adansonia digitada, Afzelia quansensis and Kirkia acuminata, and in Mecrusse is found only Androstachys johnsonii. Considering the differences observed, it is recommended that forest management considers the particularities of each ecosystem.
\end{abstract}




\section{Introdução}

Moçambique possui um grande potencial de recursos florestais e outras formações lenhosas, cobrindo uma área de aproximadamente $70 \%$ do território nacional, que correspondem a 54,8 milhões de ha (Marzoli, 2008).

A floresta nativa em Moçambique é composta de vários tipos florestais. Em Miombo, Mopane e Mecrusse estão entre as áreas mais importantes para a exploração florestal (Marzoli, 2008), não só para a satisfação das necessidades básicas das comunidades rurais, como também para o abastecimento da indústria florestal e para a exportação (Chidumayo et al., 1996).

O conhecimento da estrutura e composição desses ecossistemas é de extrema importância para a tomada de decisões referentes ao manejo florestal ou de tratamentos silviculturais a serem empregados, assim como para o desenho de planos de manejo sustentáveis (Bila \& Mabjaia, 2012).

Segundo Santos et al. (2016), a conservação de áreas florestais nativas passa necessariamente pela compreensão do seu comportamento face às perturbações naturais e antropogênicas. A partir da distribuição diamétrica, se pode conhecer as espécies que compõem a floresta, assim como o seu comportamento estrutural.

A distribuição diamétrica permite-nos também caracterizar as tipologias florestais (Reis et al., 2014), estágios de sucessões florestais, estado de conservação, regimes de manejo, processos da dinâmica de crescimento e produção, grupos ecológicos (espécies pioneiras secundárias e clímax), grupos de usos comercial, potencial e outros. É utilizada também como guia para referência de corte e, sobretudo, como verificador de sustentabilidade ambiental de manejo.

Uma distribuição diamétrica considerada adequada para qualquer objetivo de manejo de florestas nativas é aquela que apresenta um número de árvores decrescente por sucessivas classes de diâmetro (Borders et al., 1987; Vanclay, 1994; Podlaski, 2006). Tendo essa distribuição, teoricamente haverá número suficiente de árvores por cada classe de diâmetro para substituir a mortalidade natural e suprir árvores para cortes seletivos. Vários estudos têm sido desenvolvidos para descrever a distribuição diamétrica de ecossistemas de Miombo, Mopane e Mecreusse (Chamba et al., 2002; Bila et al., 2011; Bila \& Mabjaia, 2012; Mate et al., 2014; Magalhães \& Seifert, 2015). Mas, ainda são inexistentes estudos sobre funções densidade de probabilidade em Moçambique.
O presente trabalho tem como objetivo comparar o desempenho das funções densidade de probabilidade para expressar a distribuição diamétrica de Miombo Mopane e Mecrusse, que representam três diferentes ecossistemas florestais de Moçambique.

\section{Material e métodos}

O trabalho foi realizado nas províncias de Gaza para a tipologia florestal de Mopane, Inhambane para a tipologia florestal de Mecrusse e Zambézia para a tipologia florestal de Miombo.

Mopane é um tipo de formação florestal caracterizada pela predominância de Colophospermum mopane, uma espécie da família Fabaceae e subfamília Caesalpiniodeae, que ocorre naturalmente na África do Sul, Zimbábwe, Angola, Botswana, Zâmbia, Namíbia e Malawe (Nube et al., 2016; Bila et al., 2012).

Mecrusse é um tipo de vegetação que ocorre em Moçambique de forma gregária, onde a principal e única espécie presente no seu dossel é Androstachys johnsonii, pertecente à família Euphorbiaceae. Essa vegetação se caracteriza pela baixa diversidade biológica e pela uniformidade em termos de indivíduos, tanto em diâmetro como em altura. Em Moçambique é encontrada nas províncias de Gaza e Inhambane (Bila et al., 2011; Magalhães \& Seifert, 2015).

Miombo é um termo usado para descrever as áreas florestais das regiões centro, sul e leste de África, com predominância dos gêneros Brachystegia, Jubernardia e/ou Isoberlina, todos da família Fabaceae e subfamíla Caesalpinioideae. Estes ecossistemas ocorrem naturalmente nas regiões semiáridas do interior e as florestas não diferenciadas na região costeira da região central (Sitoe et al., 2012).

A província de Gaza está situada na região sul do país e as parcelas permanentes se localizam no Distrito de Mabalane. O clima segundo Köppen é classificado como BSh do tipo estepe local com invernos secos com temperaturas médias anuais de $24,2{ }^{\circ} \mathrm{C}$ e precipitação média anual de $493 \mathrm{~mm}$. Mabalane é caracterizada por três principais unidades de solos, com base principalmente na fisiografia do terreno, na textura e na cor: argilosos, localizados nas zonas baixas e nas encostas inferiores; arenosos, localizados nas zonas altas; e solos franco argilosos, onde o relevo é ligeiramente acidentado com altitudes inferiores a 200 m (Instituto Nacional de Investigação Agrária, 1999) 
Na província de Inhambane, também localizada na região sul do país, a área de estudo está situada no Distrito de Mabote. O clima da região, segundo a classificação de Köppen, é caracterizado como BSW do tipo semi-árido com duas estações, uma quente e chuvosa, de novembro a dezembro, e outra fria e seca, de janeiro a outubro. A temperatura média anual é de $24,0{ }^{\circ} \mathrm{C}$, a precipitação média anual é de $629 \mathrm{~mm}$ e a evaporação média anual é de cerca de $1.585 \mathrm{~mm}$. Mabote é constituído por solos arenosos não especificados e cerca de $12,5 \%$ da área é composta por solos de coluviões argilosos. O relevo é do tipo planície, com altitudes não superiores a $150 \mathrm{~m}$. Na região não existem montanhas nem áreas declivosas (Chamba et al., 2002).

Na província da Zambézia, região central do centro do país, o local de estudo se localiza nos Distritos da Maganja da Costa, Gilé e Morrumbala. O clima anual desta região é tropical chuvoso do tipo AW, segundo a classificação de Köppen, com temperaturas médias anuais de $25,3^{\circ} \mathrm{C}$, e precipitação média anual de $1.346 \mathrm{~mm}$. Os solos são de tipo franco argiloso avermelhado. O relevo é de forma planáltica e a topografia sobe do litoral para o interior, onde se ergue até a Serra de Namuli, com os seus 2.419 m acima do nível do mar.

Os dados foram obtidos de 16 parcelas permanentes, dos quais 10 foram estabelecidas nos ecossistemas de Miombo, 3 nos ecossistemas de Mopane e 3 nos de Mecrusse pelo Centro de Investigação Florestal do Instituto de Investigação Agrária de Moçambique. $\mathrm{O}$ tamanho das parcelas foi de $50 \mathrm{~m} \times 50 \mathrm{~m}$ para Mopane e Mecrusse e 100 m x 50 m para Miombo. Para localização dos indivíduos nas parcelas foi usado o sistema de coordenadas (X e Y).

Em cada parcela em Mopane e Mecrusse foram medidos os diâmetros a 1,30 m do solo (DAP), com fita dendrométrica, de todos os indivíduos com $\mathrm{DAP} \geq 10 \mathrm{~cm}$ e em Miombo todos com DAP $\geq 20 \mathrm{~cm}$. Também foram medidas as alturas comercial, do fuste (até a primeira bifurcação) e total de cada árvore, usando hipsômetro Sunnto.

Foram estimados o número de espécies, gêneros e famílias com base nas 16 amostras. A evolução da distribuição diamétrica foi feita para cada ecossistema, ajustando 3 modelos de função densidade de probabilidade (Weibull 3P, Gama e Beta), com base no pacote estatístico Table Curve 2D V3.1. Para cada ecossistema foi determinado o intervalo e o número de classes com base no método de Struges.
A distribuição de Weibull 3P pode assumir várias formas, de acordo com os coeficientes, e ajusta-se muito bem para os dados de florestas nativas e plantadas. Para o presente trabalho foi ajustada a função com 3 parâmetros (equação 1).

$$
\mathrm{F}(\chi)=\frac{\gamma}{\beta}\left(\frac{\mathrm{d}-\alpha}{\beta}\right)^{\gamma-1}\left[-\left(\frac{\mathrm{d}-\alpha}{\beta}\right)^{\gamma}\right]
$$

Em que: $\alpha=$ parâmetro de locação, no início da curva; $\beta$ $=$ parâmetro de escala da curva; $\gamma=$ parâmetro de forma, que define o tipo de curva; $d=$ diâmetro.

A função Gama é flexível, podendo ser aplicada em florestas nativas ou plantadas (equação 2). Pode ajustarse a diferentes tipos de curvas, podendo assumir diversos graus de assimetria (Scolforo, 1998).

$$
\mathrm{F}(\chi)=\frac{(\mathrm{d}-\mathrm{d} \min )^{\alpha-1} * \mathrm{e}^{\frac{-(\mathrm{d}-\mathrm{d} \min )}{\beta}}}{\Gamma(\alpha) * \beta^{\alpha}}
$$

Em que: $\Gamma=$ Gama, $\alpha$ e $\beta=$ parâmetros, dmax $=$ diâmetro máximo, dmin- diâmetro mínimo, $d=$ diâmetro observado.

A função Beta de distribuição contínua é também muito flexível e pode ser utilizada tanto para florestas nativas como para plantadas, podendo assumir várias formas para uma ampla faixa de distribuições (equação 3). A sua função possui limites definidos entre os menores e maiores valores observados (Scolforo, 1998).

$\mathrm{F}(\chi)=\frac{\Gamma(\alpha+\beta)}{\Gamma(\alpha) \Gamma(\beta)} * \frac{1}{(\mathrm{~d} \max -\mathrm{d} \min )^{\alpha+\beta-1}} *(\mathrm{~d}-\mathrm{d} \min )^{\alpha-1}(\mathrm{~d}-\mathrm{d} \max )^{\beta-1}$

Em que: $\Gamma=$ Beta, $\alpha$ e $\beta$ parâmetros, $d$ max $=$ diametro máximo, $d \min =$ diâmetro mínimo, $d=$ diâmetro observado.

Os testes de aderências dos modelos foram feitos com base no teste de Kolmogorov-Smirnov, que considera a maior diferença entre duas distribuições (equação 4). A significância do teste é dada pela (equação 5).

$$
\mathrm{D}=\operatorname{máximo}\left|\frac{\operatorname{Fobs}(\chi)-\operatorname{Fest}(\chi)}{\mathrm{n}}\right|
$$

Em que: $\operatorname{Fobs}(\mathrm{x})$ e $\operatorname{Fest}(\mathrm{x})=$ frequência acumulada observada e estimada para cada classe, respectivamente, $\mathrm{D}=$ ponto de maior divergência, que é o valor $\mathrm{D}$ de $\mathrm{K}-\mathrm{S}$. $\mathrm{O}$ menor $\mathrm{D}$ entre distribuições, indicará o melhor ajuste. 


$$
\operatorname{Dn}(5 \%)=\frac{1,36}{\sqrt{n}}
$$

Em que: $D n=$ O valor tabelado em nível $\alpha$ de probabilidade para $\mathrm{n}$ indivíduos, $n=$ número total de árvores.

\section{Resultados}

Em Miombo foram observadas 32 espécies distribuídas em 15 famílias com DAP $\geq 20 \mathrm{~cm}$. As espécies com maior participação foram das famílias Fabaceae, Julbernardia globiflora, Brachystegia spiciformes, Brachystegia boemii, Pterocarpus angolensis, e Combretaceae, Ptelliopsis myrtifolia. Nos ecossistemas de Mopane foram observadas 15 espécies florestais distribuídas em onze famílias botânicas com DAP $\geq 10 \mathrm{~cm}$. As famílias com maior número de espécies foram Fabaceae (duas espécies), Capparaceae (uma espécies), Bombacaceae (uma espécie), Simaroubaceae (uma espécie) e as demais famílias foram representadas por uma única espécie. As espécies mais predominantes nesses ecossistemas são Colophospermum mopane, Boscia mossambicensis, Adansonia digitada, Afzelia quansensis e Kirkia acuminata. Em Mecrusse verificou-se apenas a ocorrência de uma espécie da família Euphobiaceae (Androstachys johnsonii). As espécies com maior valor de importância, por local, estão apresentadas na Tabela 1 .

$\mathrm{Na}$ tabela 2 são apresentados os coeficientes de ajuste dos modelos e o $\mathrm{R}^{2}$ ajustado nos três ecossistemas estudados.

A função de Weibull 3P foi aderente para os três ecossistemas e as funções Gama e Beta apresentaram aderência em Miombo e Mopane (Tabela 3).

Na tabela 4 são apresentadas as frequências observadas e as estimadas por cada função, em cada centro de classe nos ecossistemas de Miombo. Mopane e Mecrusse.
Tabela 1. Índice do valor de importância, abundância e dominância nos ecossistemas de Miombo, Mopane e Mecrusse.

Table 1. Importance index, abundance and dominance values in the Miombo, Mopane and Mecrusse ecosystems

\begin{tabular}{lccc}
\hline \multirow{2}{*}{\multicolumn{1}{c}{ Espécie }} & $\begin{array}{c}\text { Índice de valor } \\
\text { de importância } \\
(\mathbf{\%})\end{array}$ & $\begin{array}{c}\text { Abundância } \\
\left(\mathbf{a r v} \mathbf{h a}^{-1}\right)\end{array}$ & $\begin{array}{c}\text { Dominância } \\
\left(\mathbf{m}^{\mathbf{2}} \mathbf{h a} \mathbf{a}^{-1}\right)\end{array}$ \\
\cline { 2 - 4 } Miombo \\
Julbernardia globiflora & 17,78 & 39,00 & 2,23 \\
Brachystegia spiciformes & 15,38 & 24,40 & 5,39 \\
Brachystegia boemii & 10,90 & 23,80 & 2,67 \\
Pterocarpus angolensis & 7,49 & 12,00 & 1,97 \\
Ptelliopsis myrtifolia & 6,31 & 6,40 & 1,70 \\
\cline { 2 - 4 } & \multicolumn{3}{c}{ Mopane } \\
\cline { 2 - 4 } Colophospermum mopane & 86,68 & 258,67 & 5,87 \\
Boscia mossambicensis & 23,38 & 6,67 & 0,10 \\
\hline Adansonia digitada & 15,21 & 2,67 & 0,90 \\
Afzelia quansensis & 12,71 & 1,33 & 0,38 \\
Kirkia acuminata & 12,14 & 6,67 & 0,21 \\
\cline { 2 - 4 } & \multicolumn{3}{c}{ Mecrusse } \\
Androstachys johnsonii & 100,00 & 723,00 & 20,62 \\
\hline
\end{tabular}

Tabela 2. Estatística e parâmetros das distribuições Beta, Gama e Weibull 3P.

Table 2. Statistics and parameters of the Beta, Gamma and Weibull 3P distributions.

\begin{tabular}{cccccccccc}
\hline Coef. & \multicolumn{3}{c}{ Par. Weibull3p } & \multicolumn{3}{c}{ Par. Gama } & \multicolumn{3}{c}{ Par. Beta } \\
\cline { 2 - 10 } & Mop & Mec & Mio & Mop & Mec & Mio & Mop & Mec & Mio \\
\hline$\alpha$ & 7,23 & 9,45 & 20,15 & 15,04 & 15,20 & 12,43 & 2,21 & 1,63 & 1,01 \\
$\beta$ & 9,30 & 6,56 & 12,20 & 1,03 & 0,95 & 2,12 & 14,20 & 7,03 & 5,85 \\
$\gamma$ & 2,24 & 1,59 & 1,08 & & & & & & \\
\hline$R^{2}$ aj. & 0,99 & 0,98 & 0,99 & 0,99 & 0,98 & 0,98 & 0,92 & 0,99 & 0,99 \\
\hline
\end{tabular}

Em que: $\alpha, \beta, \gamma=$ coeficientes; Par $=$ parâmetros; Mop = Mopane; Mec $=$ Mecrusse $;$ Miom $=$ Miombo

Tabela 3. Teste de Kolmogorov-Smirnov das funções ajustadas nos ecossistemas de Miombo, Mopane e Mecrusse.

Table 3. Kolmogorov-Smirnov test of the adjusted functions in Miombo, Mopane and Mecrusse ecosystems.

\begin{tabular}{cccccccc}
\hline \multirow{2}{*}{ FDP } & \multicolumn{2}{c}{ Miombo } & \multicolumn{2}{c}{ Mopane } & \multicolumn{2}{c}{ Mecrusse } \\
\cline { 2 - 7 } & D. calcu & D. tabel & D. calcu & D. tabel & D. calcu & D. tabel \\
\hline Weibull 3P & $0,010^{*}$ & 0,130 & $0,042^{*}$ & 0,108 & $0,010^{*}$ & 0,061 \\
Gama & $0,199 *$ & 0,130 & $0,045^{*}$ & 0,108 & 0,096 & 0,061 \\
Beta & $0,010^{*}$ & 0,130 & $0,093^{*}$ & 0,108 & 0,036 & 0,061 \\
\hline
\end{tabular}

Aderente pelo teste de Kolmogorov-Smirnov, com a probabilidade de $\alpha=$ $0,01 . \mathrm{FDP}=$ funççao densidade e probailidade 
Com a função Weibull 3P foram observados os valores estimados mais próximos dos valores observados (Figura 1a). Com a função Gama detectou-se subestimação nas primeiras classes nos ecossistemas de Miombo e Mecrusse, e também nas classes a partir de $37,5 \mathrm{~cm}$ no ecossistema de Miombo. O mesmo foi observado no ecossistema de Mopane na classe de 27,5 cm (Figura 1b).
A função Beta mostrou bons resultados de ajuste nos ecossistemas de Miombo e Mopane, segundo o teste de aderência dos modelos. Nos ecossistemas de Miombo os valores estimados estão mais próximos dos valores observados (Figura 1c). Em Mopane foi observada superestimação na primeira classe e na classe de $22,5 \mathrm{~cm}$.
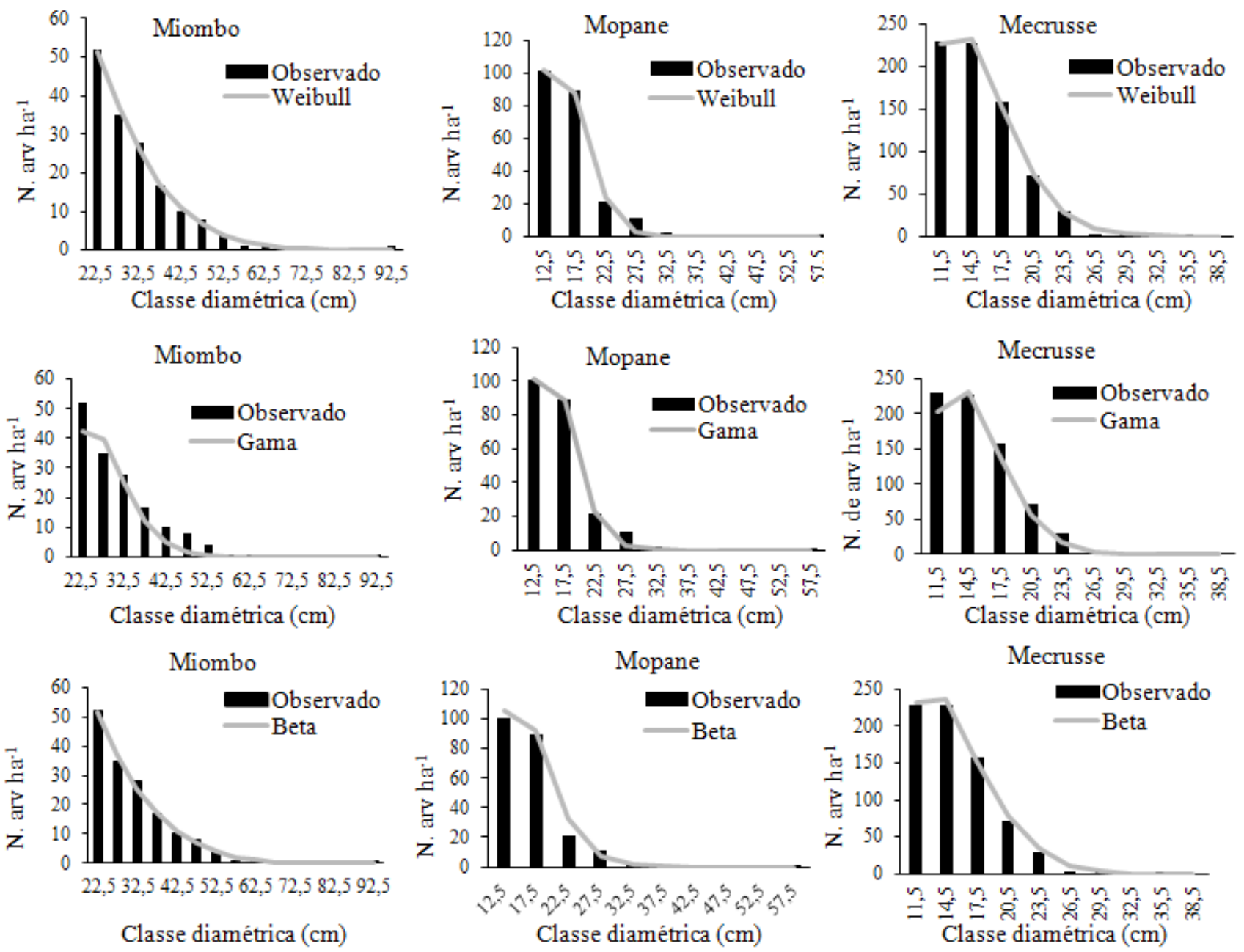

Figura 1. Distribuição diamétrica observada e histograma das funções a) Weibull 3P, b) Gama e c) Beta, ajustadas para os ecossistemas de Miombo, Mopane e Mecrusse.

Figure 1. Observed diameter distribution and function histogram of a) Weibull 3P, b) Gama and c) Beta, adjusted for Miombo, Mopane and Mecrusse ecosystems. 
Tabela 4. Frequências observadas e estimadas por cada função densidade e probabilidade nos ecossistemas de Miombo, Mopane e Macrusse.

Table 4. Observed and estimated frequencies by each function density and probability in Miombo, Mopane and Macrusse ecosystems.

\begin{tabular}{|c|c|c|c|c|}
\hline \multirow{2}{*}{$\begin{array}{l}\text { Centro de } \\
\text { classe }\end{array}$} & \multirow{2}{*}{$\begin{array}{l}\text { Frequência } \\
\text { real (ind ha') }\end{array}$} & \multicolumn{3}{|c|}{ Frequências estimadas (ind ha ${ }^{-1}$ ) } \\
\hline & & Weibull 3P & Gama & Beta \\
\hline & & \multicolumn{3}{|c|}{ Miombo } \\
\hline 22,5 & 52,0 & 51,2 & 42,2 & 51,5 \\
\hline 27,5 & 35,0 & 37,3 & 39,4 & 36,8 \\
\hline 32,5 & 28,0 & 25,9 & 25,1 & 25,4 \\
\hline 37,5 & 17,0 & 17,2 & 12,1 & 17,0 \\
\hline 42,5 & 10,0 & 10,9 & 4,8 & 11,0 \\
\hline 47,5 & 8,0 & 6,6 & 1,6 & 6,8 \\
\hline 52,5 & 4,0 & 3,9 & 0,5 & 4,0 \\
\hline 57,5 & 1,0 & 2,2 & 0,1 & 2,2 \\
\hline 62,5 & 1,0 & 1,2 & 0,0 & 1,1 \\
\hline 67,5 & 0,0 & 0,6 & 0,0 & 0,5 \\
\hline 72,5 & 0,0 & 0,3 & 0,0 & 0,2 \\
\hline 77,5 & 0,0 & 0,2 & 0,0 & 0,1 \\
\hline 82,5 & 0,0 & 0,1 & 0,0 & 0,0 \\
\hline 87,5 & 0,0 & 0,0 & 0,0 & 0,0 \\
\hline \multirow[t]{2}{*}{92,5} & 1,0 & 0,0 & 0,0 & 0,0 \\
\hline & & \multicolumn{3}{|c|}{ Mopane } \\
\hline 12,5 & 101,0 & 101,6 & 100,9 & 106,0 \\
\hline 17,5 & 89,0 & 88,3 & 88,3 & 92,8 \\
\hline 22,5 & 21,0 & 24,2 & 23,4 & 33,1 \\
\hline 27,5 & 11,0 & 2,3 & 3,0 & 7,6 \\
\hline 32,5 & 2,0 & 0,1 & 0,3 & 1,1 \\
\hline 37,5 & 0,0 & 0,0 & 0,0 & 0,1 \\
\hline 42,5 & 0,0 & 0,0 & 0,0 & 0,0 \\
\hline 47,5 & 0,0 & 0,0 & 0,0 & 0,0 \\
\hline 52,5 & 1,0 & 0,0 & 0,0 & 0,0 \\
\hline \multirow[t]{2}{*}{57,5} & 1,0 & 0,0 & 0,0 & 0,0 \\
\hline & & \multicolumn{3}{|c|}{ Mecrusse } \\
\hline 11,5 & 229,3 & 225,9 & 204,5 & 231,5 \\
\hline 14,5 & 228,0 & 232,9 & 231,2 & 235,6 \\
\hline 17,5 & 157,3 & 148,6 & 140,5 & 152,5 \\
\hline 20,5 & 72,0 & 72,4 & 55,9 & 79,5 \\
\hline 23,5 & 29,3 & 28,8 & 16,4 & 34,0 \\
\hline 26,5 & 2,7 & 9,6 & 3,8 & 11,5 \\
\hline 29,5 & 2,7 & 2,8 & 0,7 & 2,8 \\
\hline 32,5 & 0,0 & 0,7 & 0,1 & 0,4 \\
\hline 35,5 & 1,3 & 0,2 & 0,0 & 0,0 \\
\hline 38,5 & 0,0 & 0,0 & 0,0 & 0,0 \\
\hline
\end{tabular}

\section{Discussão}

Foram registradas 32 espécies, distribuídas em 15 famílias, sendo Fabacaeae a mais representativa no presente trabalho (cerca de $77 \%$ de todas as espécies). Em estudo sobre a diversidade e composição de espécies de Miombo, Gonçalves (2017) encontrou 51 espécies distribuídas em 19 famílias, com maior diversidade da família Fabaceae, representando cerca de 30\% de todas as espécies lenhosas.

Nos ecossistemas de Mopane a espécie com a maior predominância foi Colophospermum mopane, com uma participação de cerca de $85 \%$ de todas as espécies. Esta espécie ocorree de forma gregária, com poucos indivíduos de outras espécies (Bila \& Mabjaia, 2012).

Magalhães \& Seifert (2015), em estudo sobre a modelagem de biomassa de Androstachys johnsonii nos ecossistemas de Mecrusse, encontraram cobertura dessa espécie entre 80 e $100 \%$, compatível com os resultados observados (Tabela 1).

Vários estudos foram realizados visando à modelagem da distribuição diamétrica, usando diversas funções de densidade de probabilidade. Como por exemplo, Loureiro et al. (2013) e Jesus et al. (2017) constataram que as funções de Weibull 3P, Beta e Gama se destacaram no ajuste da distribuição diamétrica.

A partir de uma distribuição da variável dendrométrica, como o DAP, a altura total, a área transversal e o volume individual, podemos descrever a floresta pelo seu grau de ocupação, abundancia e dominância, assim como o número de indivíduos por cada classe de diâmetro e os respetivos volumes disponíveis em cada classe de diâmetro. Conhecendo o grau de ocupação por meio da distribuição diamétrica, prescrição de intervenções silviculturais assim como a determinação de rendimentos da floresta por cada tipo de produto desejado, se torna mais fácil e fundamental (Prodan et al., 1997).

\section{Conclusões}

No ecossistema de Miombo as espécies mais importantes foram Julbernardia globiflora, Brachystegia spiciformes, Brachystegia boemii, Pterocarpus angolensis e Ptelliopsis myrtifolia e em Mopane foram Colophospermum mopane, Boscia mossambicensis, Adansonia digitada, Afzelia quansensis e Kirkia acuminata. No ecossistema de Mecrusse, a única espécie encontrada foi Androstachys johnsonii. 

em Moçambique

Os ecossistemas de Miombo Mopane e Mecrusse apresentam distribuição diamétrica do tipo "j invertido", tendo a função de Weibull 3P apresentado melhor performance no ajuste da distribuição diamétrica nos três ecossistemas.

A distribuição diamétrica nos três ecossistemas pode ser usada como ferramenta para determinar os volumes de madeira a serem explorados e para prever a produção futura, garantindo deste modo, a elaboração de planos de manejo sustentáveis e o uso otimizado dos recursos.

\section{Referências}

Bila, J. M. et al. Efeito dos tratamentos silviculturais nos ecossistemas de mecrusse em Mabote, Província de Inhambane, Moçambique. Pesquisa Florestal Brasileira, v. 31, n. 65, p. 63-67, 2011. DOI: 10.4336/2011.pfb.31.65.63.

Bila, J. M. \& Mabjaia, N. Crescimento e fitossociologia de uma floresta com Colophospermum mopane, em Mabalane, Província de Gaza, Moçambique. Pesquisa Florestal Brasileira, v. 32, n. 71, p. 421-427, 2012. DOI: 10.4336/2012.pfb.32.72.421.

Borders, B. et al. Percentile-based distributions characterize forest stand tables, Forest Science, v. 33, p. 570-576, 1987. DOI: 10.1093/ forestscience/33.2.570.

Chamba, E. et al. Estrutura e composição do ecossistema do Mecrusse - Mabote. Maputo: CEF, 2002. 45 p.

Chidumayo, E. \& Frost, P. Population biology of miombo trees. In: Miombo in Transition: Woodlands and Welfare in Africa. Indonesia: CIFOR, 1996. p. 59-71.

Gonçalves, F. M. P. et al. Tree species diversity and composition of Miombo Woodlands in South-Central Angola: a chronosequence of forest recovery after shifting cultivation. International Journal of Forestry Research, v. 2017, Id 6202093, 13 p., 2017. DOI: $10.1155 / 2017 / 6202093$.

Instituto Nacional de Investigação Agrária. Inventário dos recursos de terra na comunidade de Gerés. Distrito de Mabalane, Maputo, 1999. 77 p. (Série terra e água).

Jesus, C. M. et al. Funções de densidade de probabilidade para estimativa das distribuições de variáveis dendrométricas em um povoamento clonal de eucalipto. Revista Espacios, v. 38, n. 16, p. 30, 2017.
Loureiro, G. H. et al. Ajuste de funções de distribuição diamétrica para um povoamento de Araucaria angustifólia (Bert.) O. Ktze antes e após desbaste. Enciclopédia Biosfera, v. 9, n. 16, p. 56-68, 2013.

Magalhães, T. M. \& Seifert, T. Biomass Modelling of Androstachys johnsonii Prain: a comparison of three methods to enforce additivity. International Journal of Forestry Research, v. 2015, n. 878402, p. 17, 2015. DOI: 10.1155/2015/878402.

Marzoli, A. Inventario florestal nacional: avaliação integrada das florestas de Moçambique. Maputo: Ministério de Agricultura, Direcção Nacional de Terras e Florestas, 2008. 98 p.

Mate, R. et al. Biomass equations for tropical forest tree species in Mozambique. Forests, v. 5, n. 3, p. 535-556, 2014. DOI:: 10.3390/ f5030535.

Nube, T. et al. Impactos socioeconômicos das plantações florestais no Niassa, Moçambique. Floresta e Ambiente, v. 23, n. 1, p. 52-60, 2016. DOI: $10.1590 / 2179-8087.038813$.

Podlaski, R. Suitability of the selected statistical distributions for fitting diameter data in distinguished development stages and phases of near-natural mixed forests in the Swietokrzyski National Park (Poland). Forest Ecology and Management, v. 236, p. 393-402, 2006. DOI: 10.1016/j.foreco.2006.09.032.

Prodan, M. et al. Mensura forestal. San José: GTZ.IICA, 1997. $586 \mathrm{p}$.

Scolforo, J. R. S. Modelagem do crescimento e da produção de florestas plantadas e nativas. Lavras: UFLA/FAEPE, 1998. 443 p.

Reis, L. et al. Dinâmica da distribuição diamétrica de algumas espécies de sapotaceae após exploração florestal na Amazônia Oriental. Revista Ciências Agrárias, v. 57, n. 3, 2014. DOI: 10.4322/ rca.ao1401.

Santos, R. et al. Distribuição diamétrica de uma comunidade arbórea na Floresta Estadual do Amapá, Brasil. Biota Amazônia, v. 6, n. 2, p. 24-31, 2016. DOI: 10.18561/2179-5746/biotaamazonia.v6n2p24-31.

Sitoe, A. et al. O contexto de REDD+ em Moçambique: causas, atores e instituições. Bogor: CIFOR, 2012. (Publicação ocasional, 76).

Vanclay J. Modelling forest growth and yield: applications to mixed tropical forests. Wallingford: CAB International, 1994. 330 p. 